\title{
No relationship between biopsy sites near the main testicular vessels or rete testis and successful sperm retrieval using conventional or microdissection biopsies in 220 non-obstructive azoospermic men
}

\author{
J Ullrich Schwarzer ${ }^{1}$, Heiko Steinfatt ${ }^{1}$, Manfred Schleyer ${ }^{2}$, Frank M Köhn ${ }^{3}$, Klaus Fiedler ${ }^{2}$, Irene von Hertwig ${ }^{2}$, \\ Gottfried Krüsmann ${ }^{2}$ and Wolfgang Würfel ${ }^{2}$
}

In 220 consecutive patients with non-obstructive azoospermia, sperm retrieval was attempted by a combination of conventional and microdissection testicular sperm extraction (TESE). For sperm retrieval, 2-3 conventional biopsies were performed followed by a microdissection TESE in cases of negative conventional biopsies. During the surgery, the vasculature of the testis was assessed using the operative microscope, and the location of positive biopsies was registered in relation to the blood supply. The overall sperm retrieval rate was $58.2 \%$. From the initial conventional biopsies, sperm could be retrieved in $46.8 \%$ of the patients. With microdissection TESE, sperm could be retrieved from an additional $11.4 \%$ of the patients. The further use of microdissection TESE improved the sperm retrieval rate significantly $(P=0.017)$. No significant accumulation of positive biopsies was found towards the rete testis or the main testicular vessels.

Asian Journal of Andrology (2013) 15, 795-798; doi:10.1038/aja.2013.98; published online 9 September 2013

Keywords: intracytoplasmic sperm injection; male infertility; microdissection testicular sperm extraction; non-obstructive azoospermia; sperm retrieval

\section{INTRODUCTION}

The retrieval of testicular sperm combined with intracytoplasmic sperm injection (ICSI) has been established as a standard treatment option for patients with non-obstructive azoospermia (NOA). Whereas sperm retrieval poses no difficulties in patients with obstructive azoospermia, sperm extraction is a challenge in patients with NOA. NOA comprises different clinical entities, different aetiologies and different histological patterns. Competing methods of testicular sperm retrieval are used: e.g., fine needle aspiration, multiple random biopsies and microdissection testicular sperm extraction (M-TESE). Sperm retrieval rates for patients with NOA range from 30\% to $70 \%$ and depend on the method of retrieval, the patient selection, the number of biopsies and the amount of removed tissue, among other factors. Although evidence supports the advantages of M-TESE, the discussion of the best method for sperm retrieval in NOA is ongoing. ${ }^{1}$ Methods that are able to predict the potential success-rate of sperm retrieval and methods that can provide information about the location of the spermatogenesis would be of great value to further improve the treatment of men with NOA. The prognostic value of the existing clinical parameters (e.g., follicle-stimulating hormone, testis volume, etc.) is unsatisfactory, as only histology seems to correlate considerably well with retrieval rates. The findings of Silber $e t$ al. $^{2}$ suggested that the distribution of spermatogenesis in the testis among patients with NOA must be diffusely multifocal and occur relatively homogenously throughout the testicle. However, small studies have suggested a correlation between testicular perfusion and focal spermatogenesis. ${ }^{3,4}$ In a study of 12 patients with NOA, sperm quality was highest in areas of high tissue perfusion, as determined by Doppler ultrasound and laser Doppler flowmetry. ${ }^{5}$ Schlegel observed that sperm-containing regions can be identified microsurgically, as seminiferous tubules that contain many developing germ cells, rather than Sertoli cells alone, are likely to be larger and more opaque than tubules without sperm production. ${ }^{6}$ Hence, Schlegel introduced the concept of M-TESE, which also allows for a detailed visualisation of the vascular supply of the testis. ${ }^{6}$ In the present study, we sought to define whether a relationship between the central testicular vasculature and focal spermatogenesis can be confirmed by M-TESE or whether sperm foci are instead distributed sparsely but evenly, as the findings of Silber $e t a l^{2}$ suggested.

\section{MATERIALS AND METHODS}

Sperm retrieval by M-TESE was performed in 220 consecutive patients with NOA in our institution between 2007 and 2013. The diagnosis of NOA was based on clinical findings, including medical history, physical examination, scrotal and transrectal ultrasounds, serum

${ }^{1}$ Andrologie Centrum München, München D-81241, Germany; ${ }^{2}$ Kinderwunsch Centrum München, München D-81241, Germany and ${ }^{3}$ Andrologicum München, München D-80331, Germany 


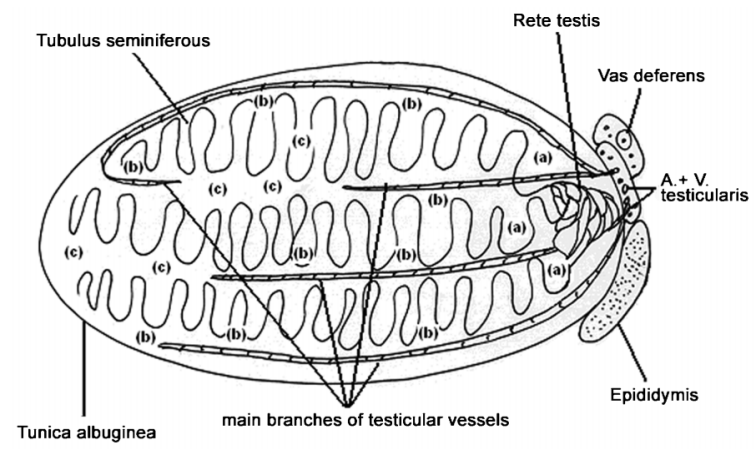

Figure 1 Location of testicular biopsies in relation to the main blood supply. (a) is location next to the rete testis; (b) is location next to the main branches of the testicular vessels; (c) is location distant from the main branches of the testicular vessels.

follicle-stimulating hormone, karyotype analysis and azoospermia factor testing. At least two semen analyses had to have shown azoospermia, according to the WHO criteria. ${ }^{7}$ The study followed the ethical guidelines established for human subjects by the Department of Urology of the Technische Universität München.

For sperm retrieval, a midline incision was made in the scrotum under general anaesthesia. Starting with the testis with the larger volume, three conventional biopsies were taken in the peripheral avascular regions at the upper and lower poles and also in the midportion of the testis, if the testicular volume was found to be above $5 \mathrm{ml}$. If the testicular volume was below $5 \mathrm{ml}$, only two conventional biopsies were taken. An additional small sample of testicular tissue was taken from the upper pole for histological examination. If no sperm could be found by using the conventional biopsies, the midportion of the tunica albuginea was widely and equatorially opened in an avascular region, and additional biopsies were performed according to Schlegel's ${ }^{6}$ principles of M-TESE. All of the samples (conventional and microsurgical) were immediately analysed by the embryologist. If sperm suitable for ICSI could be found, the material was cryopreserved, and the operation was complete. If no sperm could be found, the surgery was continued in an analogous manner on the contralateral side. During the surgery, each biopsy was characterized by the surgeon into three categories: (i) close to the rete testis; (ii) close to main vessels; or (iii) distant from the main blood supply. This characterisation takes advantage of the detailed visualisation of the vascular supply that is possible through the microsurgical technique. Figure 1 shows a schematic representation of the testicular architecture with respect to the main blood supply.

Figure 2 shows the intraoperative site during microdissection TESE. Dilated and opaque seminal tubules suggest the presence of the focal areas of spermatogenesis. After the testicular lobes have been divided using a vessel-sparing microsurgical technique, the main blood supply of the testis is clearly visualized.

\section{Statistical analysis}

Microsoft Excel 2007 (Microsoft, Redmond, WA, USA) and WinSTAT for Excel (R. Fitch Software, Staufen, Germany) were used to perform all statistical calculations. Chi-square analysis was used to compare conventional TESE with conventional TESE and additional M-TESE; a Chi-square test of independence was used to compare the different sperm positive locations; and the relationship of retrieval rates by initial conventional biopsies to the additional retrieval rates by M-TESE was described by Pearson's correlation coefficient. $P<0.05$ was considered to be statistically significant.

\section{RESULTS}

The overall sperm retrieval rate was $58.2 \%$. From the initial conventional biopsies, sperm were retrieved in $46.8 \%$ of the patients. With MTESE, sperm were retrieved from an additional $11.4 \%$ of patients. The additional use of M-TESE improved the sperm retrieval rate significantly ( $P=0.017$; Table 1). A negative correlation was found between the retrieval rate in the initial biopsies and the additional retrieval rate by M-TESE depending on histology (Pearson's correlation coefficient $r=-0.87, P=0.0276$ ).

The sperm-positive samples that were retrieved with our approach had a highly significant tendency to be located at a distance from the main testicular blood supply when the entire cohort was considered $(P<0.0001$; Figure 3a). When only the distribution of the spermpositive samples that were retrieved by M-TESE was considered (Table 2), no significant accumulation was found towards a particular location $(P=0.0994$; Figure $3 \mathbf{b})$.
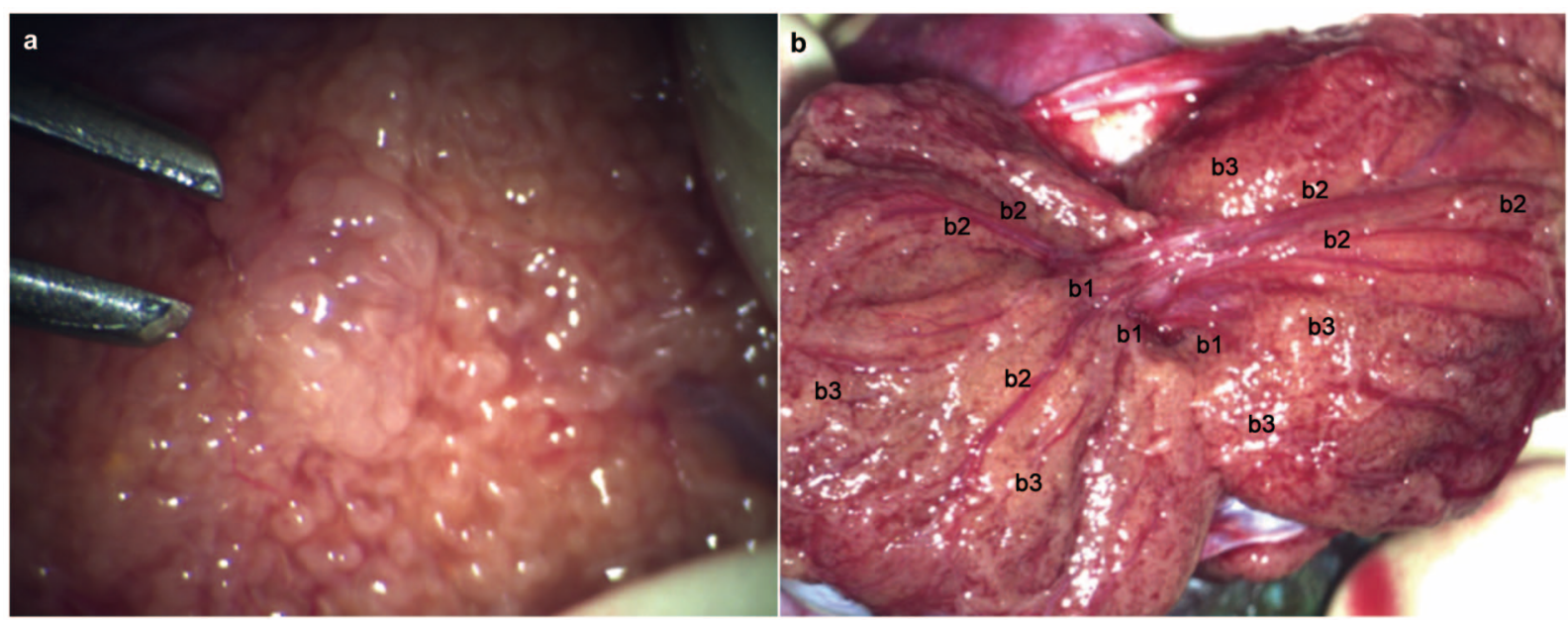

Figure 2 (a) Dilated and opaque seminiferous tubule suggesting a focal area of spermatogenesis; (b) intraoperative view of the divided testicular lobes using a vesselsparing microsurgical technique: b1 is location next to the rete testis, b2 is location next to the main branches of testicular vessels and b3 is location distant from the main branches of the testicular vessels. 
Table 1 Sperm retrieval rates for patients with NOA using conventional TESE combined with microdissection TESE

\begin{tabular}{|c|c|c|c|c|c|c|c|}
\hline Histology & $\begin{array}{c}\text { Patients } \\
\text { (n) }\end{array}$ & $\begin{array}{c}\text { Overall sperm- } \\
\text { positive }(\mathrm{n})\end{array}$ & $\begin{array}{c}\text { Overall sperm- } \\
\text { positive (\%) }\end{array}$ & $\begin{array}{c}\text { Convent. sperm- } \\
\text { positive (n) }\end{array}$ & $\begin{array}{c}\text { Convent. sperm- } \\
\text { positive (\%) }\end{array}$ & $\begin{array}{c}\text { M-TESE sperm- } \\
\text { positive (n) }\end{array}$ & $\begin{array}{c}\text { M-TESE sperm- } \\
\text { positive (\%) }\end{array}$ \\
\hline scos & 75 & 24 & 32.0 & 12 & 16.0 & 12 & 16.0 \\
\hline TubSc & 33 & 11 & 33.3 & 7 & 21.2 & 4 & 12.1 \\
\hline MatArr & 30 & 20 & 66.7 & 17 & 56.7 & 3 & 10.0 \\
\hline MixAtr & 42 & 40 & 95.2 & 36 & 85.7 & 4 & 9.5 \\
\hline Hyposp & 26 & 26 & 100.0 & 26 & 100.0 & 0 & 0.0 \\
\hline Other $^{a}$ & 14 & 7 & 50.0 & 5 & 35.7 & 2 & 14.3 \\
\hline Sum & 220 & 128 & 58.2 & 103 & 46.8 & 25 & 11.4 \\
\hline
\end{tabular}

Abbreviations: Hyposp, hypospermatogenesis; MatArr, maturation arrest; MixAtr, mixed atrophy; M-TESE, microdissection testicular sperm extraction; NOA, non-obstructive azoospermia; SCOS, Sertoli cell-only syndrome; TESE, testicular sperm extraction; TubSc, tubular sclerosis.

${ }^{a}$ Other/not classified.

\section{DISCUSSION}

Testicular sperm retrieval in combination with ICSI has revolutionized the therapeutic options for patients with NOA. Nevertheless, sperm retrieval remains unsuccessful in many patients with NOA, despite considerable efforts. The ability to locate even sparse foci of spermatogenesis in the testis is a key issue for a successful retrieval strategy that goes beyond conventional TESE. Different methods have been applied to facilitate this task: e.g., Doppler ultrasound, ${ }^{3-5}$ laser flowmetry,${ }^{5}$ magnetic resonance spectroscopy ${ }^{8}$ optical coherence tomography, ${ }^{9}$ confocal fluorescence microscopy ${ }^{10,11}$ and multiphoton tomography. ${ }^{12}$ The spatial resolution, penetration depth and need for extrinsic labels or dyes, such as sperm-specific antibodies, vary greatly between these imaging modalities and might limit their use in clinical practice. The potential damage to spermatozoa or testicular tissue caused by these different modalities depends on the energy levels needed for molecular excitation, as well as the type and application of extrinsic labels, among other factors. Thus far, several large studies have shown that only M-TESE has the potential to identify focal areas of spermatogenesis during sperm retrieval in a clinical setting. ${ }^{6,13-15}$ In one series of 12 patients with NOA, Doppler ultrasound and laser flowmetry were used to guide the sperm retrieval and to isolate sperm suitable for ICSI in $75 \%$ of patients. ${ }^{5}$ In contrast, the findings of Silber et al. ${ }^{2}$ suggested that sparse sperm foci are distributed equally throughout the testis in patients with NOA without any correlation to the testicular blood supply.

In the present study, we sought to define whether a relationship between the central vasculature of the testis and the location of sperm foci could be confirmed by M-TESE. With the operative microscope, the blood supply of the testis can be well visualized. Therefore, in addition to the potential to improve the sperm yield, the ability to
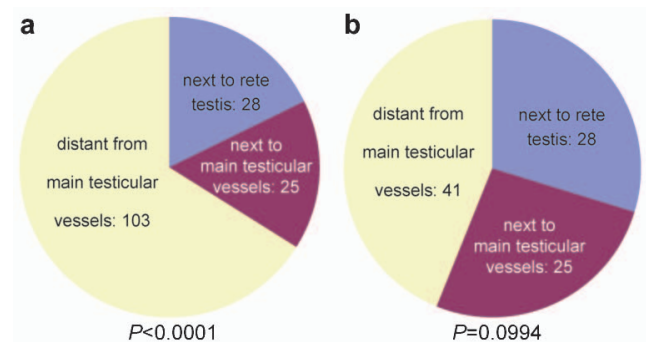

Figure 3 Distribution of sperm-positive areas in relation to the central testicular blood supply. (a) Initial conventional TESE combined with M-TESE; (b) distribution of sperm foci that were detected exclusively by microdissection TESE. M-TESE, microdissection testicular sperm extraction; TESE, testicular sperm extraction. detect and avoid testicular vessels can be considered a major advantage of M-TESE. ${ }^{6}$ In our current series, 220 patients with NOA underwent an initial conventional TESE with $2-3$ biopsies in a peripheral avascular area. If these biopsies yielded no sperm, the whole testicular parenchyma was searched with the operative microscope for focal spermatogenesis according to the M-TESE technique described by Schlegel. The overall sperm retrieval rate in our cohort was $58.2 \%$ with the combined technique. M-TESE yielded a further $11.4 \%$ in addition to conventional TESE. The complementary use of M-TESE leads to a significantly higher sperm yield $(P=0.017)$, as Marconi et $a . .^{15}$ also showed in their series. Depending on the histological pattern, the usefulness of M-TESE increased with decreasing conventional retrieval rates. For example, in the case of Sertoli cell-only syndrome (SCOS), the retrieval rate was doubled by additional M-TESE (retrieval rate with conventional TESE: 16\%; additional retrieval rate by M-TESE: $16 \%$ ), whereas in the case of hypospermatogenesis, sperm were found with conventional TESE in $100 \%$ of the patients. Starting with 2-3 single conventional biopsies instead of starting with M-TESE immediately helped us to avoid further surgical effort (e.g., wide equatorial opening of tunica albuginea and dissection of testicular lobes) in $46.8 \%$ of our 220 patients. Thus, the combination of conventional TESE and M-TESE may reduce morbidity in these cases. It is important to note that sperm retrieval rates depend not only on the surgical technique, but also heavily on the patient selection. In our previous series of 414 patients with NOA, 13\% had a histological diagnosis of SCOS. With conventional TESE (3-10 biopsies), the retrieval rate was $60 \% .{ }^{16}$ In our current cohort, $49 \%$ of the 220 patients had a histological diagnosis of SCOS or tubular sclerosis, and the overall sperm retrieval rate was $58 \%$, despite the additional use of M-TESE. Compared with our previous cohort from 2003, we encountered a substantial increase in unfavourable prognostic factors in our patients. For example, the percentage of patients who had already undergone TESE without success was $23 \%$ in our current cohort.

The distribution of sperm-positive samples that were retrieved by our combined approach had a highly significant tendency towards being located distant from the main testicular blood supply. As conventional biopsies were retrieved only in peripheral avascular locations of the testis, there clearly was a surgical bias towards locations distant from the main testicular blood supply which occurred in our approach to sperm retrieval. However, when only patients whose sperm was able to be retrieved by M-TESE alone were considered, no significant correlation was found between the main testicular blood supply and the sperm-positive locations. An accumulation of sperm-positive samples towards the main testicular blood supply could not be confirmed in our patients. To our knowledge, we are presenting the first systematic study that correlates intraoperative 
Table 2 Location of sperm foci that were detected only by microdissection TESE

\begin{tabular}{|c|c|c|c|c|c|c|}
\hline Histology & $\begin{array}{c}\text { Patients } \\
\text { (n) }\end{array}$ & $\begin{array}{c}\text { M-TESE } \\
\text { sperm positive } \\
\text { (n) }\end{array}$ & $\begin{array}{c}\text { M-TESE } \\
\text { sperm-positive } \\
\text { (\%) }\end{array}$ & $\begin{array}{l}\text { Peripheral positive } \\
\text { samples } \\
\text { (n) }\end{array}$ & $\begin{array}{l}\text { Intermediate positive } \\
\text { samples } \\
\text { (n) }\end{array}$ & $\begin{array}{c}\text { Central positive } \\
\text { samples } \\
\text { (n) }\end{array}$ \\
\hline TubSc & 33 & 4 & 12.1 & 6 & 4 & 4 \\
\hline MatArr & 30 & 3 & 10.0 & 11 & 5 & 5 \\
\hline MixAtr & 42 & 4 & 9.5 & 12 & 10 & 6 \\
\hline Sum & 220 & 25 & 11.4 & 41 & 25 & 28 \\
\hline
\end{tabular}

Abbreviations: Hyposp, hypospermatogenesis; MatArr, maturation arrest; MixAtr, mixed atrophy; M-TESE, microdissection testicular sperm extraction; SCOS, Sertoli cellonly syndrome; TESE, testicular sperm extraction; TubSc, Tubular sclerosis.

${ }^{\text {a } O t h e r / n o t ~ c l a s s i f i e d . ~}$

findings concerning the major testicular blood supply during M-TESE with the location of sperm-positive biopsies. Our findings conflict with the results of other groups that found a relationship between blood flow, as measured by ultrasound, and sperm-positive biopsies in small cohorts of patients with NOA. ${ }^{3-5}$ As we did not use Doppler ultrasound or laser Doppler flowmetry but instead assessed testicular vascularisation through an operative microscope, these conflicting results might be explained by different methodologies. However, our findings concur with the assessment of Ramasamy et al. ${ }^{17}$ Based on the experience of 900 men who underwent M-TESE for NOA between 2000 and 2010, Ramasamy et al. ${ }^{17}$ believe that a close correlation does not exist between testicular blood flow and the areas of spermatogenesis in patients with NOA, although this correlation was not specifically studied during their investigations.

\section{CONCLUSIONS}

In patients with NOA, the sperm retrieval rates with conventional TESE can be improved significantly when M-TESE is also performed in cases of negative conventional biopsies. The usefulness of additional M-TESE increases with decreasing conventional retrieval rates and correlates with the histological diagnosis. The combination of conventional TESE and M-TESE helped to avoid the surgical effort that accompanies M-TESE in $46.8 \%$ of our patients with NOA; therefore, a combined approach of conventional TESE and M-TESE might further reduce the morbidity of sperm retrieval. As, in $46.8 \%$ of our patients with NOA, sperm had already been found by a conventional peripheral biopsy, the combination of conventional TESE and MTESE yielded sperm-positive samples predominantly in the seminiferous tubules that were located distant from the main testicular blood supply. In the subgroup in which conventional TESE yielded no sperm and sperm-positive samples were only retrieved by M-TESE, no significant accumulation of sperm-positive samples could be found towards the main testicular vessels or towards the rete testis. The results of other authors who described a relationship between testicular perfusion and sperm-positive areas could not be confirmed in our series by using M-TESE.

\section{AUTHOR CONTRIBUTIONS}

JUS and HS contributed to conception, design, acquisition, analysis and interpretation of data, drafting the manuscript, critical revision for important intellectual content and final approval of the version to be published. MS contributed to acquisition, analysis and interpretation of data and critical revision for important intellectual content.
FMK contributed to acquisition, analysis and interpretation of data. KF, IH, GK and WW contributed to acquisition of data.

\section{COMPETING FINANCIAL INTERESTS}

All authors declare no competing financial interests.

1 van Peperstraten A, Proctor ML, Johnson NP, Philipson G. Techniques for surgical retrieval of sperm prior to ICSI for azoospermia. Cochrane Database Syst Rev 2006; CD002807.

2 Silber SJ, Nagy Z, Devroey P, Tournaye H, van Steirteghem AC. Distribution of spermatogenesis in the testicles of azoospermic men: the presence or absence of spermatids in the testes of men with germinal failure. Hum Reprod 1997; 12: 2422-8.

3 Har-Toov J, Eytan O, Hauser R, Yavetz H, Elad D et al. A new power Doppler ultrasound guiding technique for improved testicular sperm extraction. Fertil Steril 2004; 81: 430-4.

4 Herwig R, Tosun K, Pinggera GM, Soelder E, Moeller KT et al. Tissue perfusion essential for spermatogenesis and outcome of testicular sperm extraction (TESE) for assisted reproduction. J Assist Reprod Genet 2004; 21: 175-80.

5 Herwig R, Tosun K, Schuster A, Rehder P, Glodny B et al. Tissue perfusion-controlled guided biopsies are essential for the outcome of testicular sperm extraction. Fertil Steril 2007; 87: 1071-6.

6 Schlegel PN. Testicular sperm extraction: microdissection improves sperm yield with minimal tissue excision. Hum Reprod 1999; 14: 131-5.

7 World Health Organization. WHO Laboratory Manual for the Examination and Processing of Human Semen. 5th ed. Geneva: World Health Organization; 2010. p271.

8 Aaronson DS, Iman R, Walsh TJ, Kurhanewicz J, Turek PJ. A novel application of ${ }^{1} \mathrm{H}$ magnetic resonance spectroscopy: non-invasive identification of spermatogenesis in men with non-obstructive azoospermia. Hum Reprod 2010; 25: 847-52.

9 Ramasamy R, Sterling J, Manzoor M, Salamoon B, Jain M et al. Full field optical coherence tomography can identify spermatogenesis in a rodent Sertoli-cell only model. J Pathol Inform 2012; 3: 4.

10 Greenhalgh JR, Griffith TS, Wald M. The use of immunofluorescence in microdissection testicular sperm extraction. J Androl 2009; 30: 548-51.

11 Smith RP, Lowe GJ, Kavoussi PK, Steers WD, Costabile RA et al. Confocal fluorescence microscopy in a murine model of microdissection testicular sperm extraction to improve sperm retrieval. J Urol 2012; 187: 1918-23.

12 Najari BB, Ramasamy R, Sterling J, Aggarwal A, Sheth S et al. Pilot study of the correlation of multiphoton tomography of ex vivo human testis with histology. J Urol 2012; 188: 538-43.

13 Tsujimura A. Microdissection testicular sperm extraction: prediction, outcome, and complications. Int J Urol 2007; 14: 883-9.

14 Turunc T, Gul U, Haydardedeoglu B, Bal N, Kuzgunbay B et al. Conventional testicular sperm extraction combined with the microdissection technique in nonobstructive azoospermic patients: a prospective comparative study. Fertil Steril 2010; 94: 2157-60.

15 Marconi M, Keudel A, Diemer T, Bergmann M, Steger K et al. Combined trifocal and microsurgical testicular sperm extraction is the best technique for testicular sperm retrieval in 'low-chance' nonobstructive azoospermia. Eur Urol 2012; 62: 713-9.

16 Schwarzer JU, Fiedler K, Hertwig I, Krüsmann G, Würfel W et al. Male factors determining the outcome of intracytoplasmic sperm injection with epididymal and testicular spermatozoa. Andrologia 2003; 35: 220-6.

17 Ramasamy R, Reifsnyder JE, Husseini J, Eid PA, Bryson C et al. Localization of sperm during microdissection testicular sperm extraction in men with nonobstructive azoospermia. J Urol 2013; 189: 643-6. 